\title{
Cardiac Surgery in Nonagenarians: Rethinking operative strategies in the era of TAVR and TMVR
}

\author{
Sara Pereira ${ }^{1}$ \\ ${ }^{1}$ University of Utah Health
}

March 3, 2022

\begin{abstract}
As growth of the elderly population continues to increase alongside improvements in percutaneous and minimally invasive interventions, cardiac surgeons question the role of high-risk surgery in treating these patients. TAVR has transformed the management of symptomatic severe aortic stenosis in elderly patients, and has become standard therapy for patients greater than 80 years of age. With improvements in procedural risks and technical complications for both transcatheter valves and percutaneous interventions, should we rethink the operative strategies for octogenarians and nonagenarians, particularly as they apply to concurrent high-risk operations?
\end{abstract}

\section{Introduction}

Dr. Elsisy and colleagues present a comprehensive retrospective review of all patients $(32,421)$ over a 26 -year period at the Mayo clinic that underwent open heart surgery. They compared outcomes for nonagenarians and octogenarians, with a regression analysis to evaluate predictors of late mortality in nonagenarians. They determined that $0.4 \%$ (143) were nonagenarians and $12.6 \%$ (4077) of patients were octogenarians. Of nonagenarians, surgical operations were CABG at 15.7\%, CABG-Valve at 39.6\%, and isolated Valve at 44.8\%. Operative mortality was similar between nonagenarians (6\%) and octogenarians (4.6\%) and hospital complications were comparable between the two groups. While this patient cohort is a highly selected population of nonagenarians and octogenarians with an overall preoperative STS predicted mortality of $1.6 \%$ and $1.9 \%$ respectively, their operative mortality of $6 \%$ is the lowest reported mortality for nonagenarians over the past two decades. As TAVR has emerged in the recent era between 2011 and 2019, the incidence of SAVR in the Mayo group of nonagenarians and octogenarians declined to $0.3 \%$, with all of those SAVRs done in 2011. This brought operative mortality of SAVR down to 4\% from 1993 to 2011, with comparable TAVR mortality of $1 \%$ from 2011 to 2019 in reported group. The STS-PROM risk scores underrepresented the 30-day mortality in nonagenarians in this patient cohort.

\section{Discussion}

Studies regarding surgical outcomes of CABG and Valve operations in nonagenarians are rare since the adoption of TAVR and transcatheter mitral valve repair. When given the choice between percutaneous coronary interventions and staged transcatheter valve replacement versus open concurrent operation, patients and surgeons opt for minimally invasive approaches. Elsisy and colleagues present the first surgical outcomes study in nonagenarians since 2014 with exceptional results. Davis et al analyzed 61,303 patients from the Virginia statewide STS adult cardiac database, with 108 nonagenarians undergoing cardiac surgery over an 11-year period from 2002 to 2012. CABG was the most common operation in this cohort at $39.8 \%$ followed by aortic valve replacement (35.2\%), and AVR + CABG (23.1\%). Mortality was $13 \%$ for nonagenarians and highest for AVR. STS PROM underestimated true mortality with observed to expected (O:E) ratios for mortality ranging from 1.45 to 2.65 annually. ${ }^{1}$ Caceros et al reported their experience with cardiac surgery 
in nonagenarians over a 28-year period (1983 to 2011) from the Cedars Sinai database and evaluated both survival and quality of life. The 30-day overall operative mortality was $13.6 \%$, and ranged from $8.8 \%, 12.8 \%$, and $18.9 \%$ in the respective CABG-only, valve-only, and CABG-valve groups. Age, reoperation, and prior stroke were predictors of mortality. Patients however reported an $83 \%$ improvement in 12 -month quality of life in this study. ${ }^{2}$ Bridges et al identified a multivariate logistic regression model to examine predictors of operative mortality for nonagenarians and centenarians. For CABG-only patients, operative mortality was $11.8 \%$ and the major preoperative risk factors for operative mortality for CABG were emergent/salvage status, preoperative intra-aortic balloon pump, renal failure, and peripheral vascular disease or cerebrovascular disease. Operative mortality decreased to $7.2 \%$ if nonagenarians and centenarians lacked these four above risk factors. ${ }^{3}$ How do surgical outcomes compare to those of transcatheter and percutaneous procedures?

The success of TAVR in elderly patients has been closely evaluated since Dr. Alain Cribier implanted the first TAVR in France in 2002. The initial TAVI PARTNER trial in the United States enrolled 358 patients with at least high surgical risk; mean age was 83 and STS-PROM score was $11.2 \%$. In TAVI group, 30-day mortality was $5 \%$ and one-year mortality was $30.7 \%$ vs $49.7 \%$ in standard therapy group (p<0.001). ${ }^{4}$ The PARTNER-I trial subsequently enrolled 531 high risk patients in the United States with a mean age of 93 years. Within the transfemoral TAVR group, 30-day, 1-year, and 3-year mortality was 4\%, 22\%, and 48\% respectively. ${ }^{5}$ Analyzing STS/ACC TVT registry data from 2011 to 2014, Arsalan et al showed that 3,773 nonagenarians underwent TAVR with significantly higher 30 -day mortality at $8.8 \%$ vs $5.9 \%(\mathrm{p}<0.001)$, and one year mortality at $24.8 \%$ vs $22.0 \%(\mathrm{p}<0.001) .{ }^{6}$ In the international CENTER collaboration, 882 nonagenarians underwent TAVR over an eleven-year period (2007 to 2018) with a nearly two-fold higher 30-day mortality rate of $9.9 \%$ vs $5.4 \%$ ( $<<0.001)$. Nonagenarians had a higher preoperative STS-PROM risk score of $9.9 \%$ versus $6.1 \%(\mathrm{p}<0.001)$, higher logistic EuroSCORE of $20.2 \%$ vs $14.4 \%(\mathrm{p}<0.001)$, and lower EuroSCORE II of $5.0 \%$ vs $3.9 \%(\mathrm{p}<0.001) .{ }^{7}$ The STS-PROM risk score correctly predicted mortality risk for TAVR in above studies, with the logistic EuroSCORE over-estimating mortality and the newer EuroSCORE II underestimated mortality in nonagenarians.

Although percutaneous mitral devices offer promise for patients at high and prohibitive surgical risk, data is limited for elderly patients. Percutaneous mitral valve repair for heart failure patients with functional mitral regurgitation (COAPT trial) resulted in fewer heart failure hospitalizations and lower mortality at 36 months as compared with goal directed medical therapy. Median age of patients was 74 and three-year all-cause mortality in MitraClip arm was $42.8 \%$ vs $55.5 \%$ for GDMT( $\mathrm{p}=0.001) .{ }^{8}$ Transcatheter mitral valve replacement with Tendyne (Abbott) and Intrepid (Medtronic) valves offers promise for high-risk patients with mitral regurgitation with 30-day mortality of $6 \%$ in the Tendyne study and $14 \%$ with Intrepid device. At 2 years, all-cause mortality for patients receiving the Tendyne valve was $39 \%$, and $43.6 \%$ of deaths occurred during the first 90 days. Both of these devices require trans-apical implantation. ${ }^{9-11}$ Trials comparing TMVR to open surgery are currently underway with the APOLLO trial with the Tendyne device and SUMMIT trial with the Intrepid device. Experiences with TMVR using the TAVR Sapien device have been less promising for "valve in valve" (VIV), "valve in ring" (VIR) and "valve in mitral annular calcification" (VIMAC). There has been a $94 \%$ technical success rate with VIV TMVR, however technical success of VIR and VIMAC is poor at $80 \%$ and $62 \%$. Observed 30-day mortality correlates at $6 \%, 10 \%$, and $35 \%$ for VIV, VIR, and VIMAC respectively. ${ }^{12}$ Mack et al recently released the STS/ACC TVT report on transcatheter mitral valve therapy in the United States. This includes 37,475 patients undergoing mitral transcatheter procedures, predominantly transcatheter edge to edge repairs (TEER) in $90 \%$ of patients. Patients undergoing TEER had an overall mean age of 80 , median STS PROM score of $5.35 \%$, and were determined to have prohibitive surgical risk for mitral valve repair. Since 2014, mitral device implantation annually continues to grow, with overall 30-day mortality of $4.2 \%$ for TEER and $6.7 \%$ for TMVR. One-year mortality remains significant at $16.4 \%$ and $16.3 \% .^{13}$

While percutaneous coronary intervention (PCI) can be technically successful in nonagenarians, elderly patients carry increased risk for stent thrombosis, cardiogenic shock, bleeding and vascular complications, myocardial infarction, reintervention, and mortality. Consideration for PCI in nonagenarians requires careful consideration with compliance to aspirin and P2Y12 therapy and the complexity of anatomy. Results of PCI 
show high variability in patient selection, presentation, and outcomes. Evaluation of over 18 studies and 1082 patients shows 30 -day procedural mortality ranging from $0 \%$ to $34 \% \cdot{ }^{14-17}$ Recent mid-term outcomes from 880 consecutive nonagenarian patients undergoing percutaneous coronary intervention in the J-PCI OUTCOME registry shows one year mortality of $13.5 \%$ with nonagenarians showing 1.5 times higher event rates for major adverse cardiovascular events (MACE) than octagenarians. ${ }^{18}$ Of 680 consecutive nonagenarians presenting with acute coronary syndrome, overall mortality rates were $17 \%$ in-hospital and $39 \%$ at one-year follow up. Despite high mortality, PCI was independently associated with a decreased risk of 1-year all cause death, as compared with medical treatment alone. ${ }^{19}$ While short and midterm results for PCI with staged TAVR are currently unknown, we expect additive procedural risks for combined procedures in nonagenarians.

\section{Conclusion}

With future growth of transcatheter technologies for aortic, mitral, and tricuspid valve pathologies, cardiac operations in elderly patients will continue to become more complex. Above studies prove the importance of preoperative risk assessment, frailty testing, functional status, and structural heart team approach for percutaneous, transcatheter, and open cardiac operations. Furthermore, they highlight the high inherent risk of performing any intervention on extreme elderly patients. Future operations will involve coronary revascularization for complex anatomic lesions with concomitant valvular interventions and/or valvular interventions not suitable for available percutaneous devices. Furthermore, studies have shown variable accuracy in the correct prediction of surgical risk in nonagenarian patients with the STS-PROM, EUROSCORE I and newer EuroSCORE II models. Furthermore, individualized patient and family discussions with palliative care teams are instrumental in clearly delineating postoperative expectations and goals of care which aids the decision-making process.

\section{References}

1. Davis JP, LaPar DJ, Crosby IK, et al. Nonagenarians undergoing cardiac surgery. J Card Surg 2014. 29(5):600-604.

2. Caceros M., Cheng W, De Robertis M, et al, Survival and Quality of Life for nonagenarians after cardiac surgery. Ann Thor Surg 2013. 95(5): 1598-1602.

3. Bridges CR, et al. Cardiac surgery in nonagenarians and centenarians. J ACS 2003. 197(3):347-356.

4. Leon MB, Smith CR, Mack MJ, et al. Transcatheter Aortic Valve Implantation for Aortic Stenosis in patients who cannot undergo surgery. N Engl J Med 2010. 363(17):1597-1607.

5. Thourani VH, Jensen HA, Babaliaros V, et al. Outcomes in Nonagenarians undergoing Transcatheter Aortic Valve Replacement in the PARTNER-I Trial. Ann Thorac Surg 2015. 100(3):785-792.

6. Arsalan M, Szerlip M, Vemulapalli S, et al. Should Transcatheter Aortic Valve Replacement be performed in nonagenarians? Insights from the STS/ACC TVT Registry. JACC 2016. 67(12):1387-95.

7. Vlastra W, Chandrasekhar J, Vendrik J, et al. Transfemoral TAVR in Nonagenarians: From the CENTER Collaboration. JACC: Cardiovascular Interventions 2019. 12(10):911-920.

8. Mack MJ, Lindenfield J, Abraham WT, et al. 3-Year Outcomes of Transcatheter Mitral Valve Repair in patients with heart failure. JACC 2021. 77(8):1029-1040.

9. Sorajja P, Moat N, Badhwar V, et al. Initial Feasibility Study of a New Transcatheter Mitral Prosthesis: The First 100 Patients. JACC 2019. 73(11): 1250-1260.

10. Bapat V, Rajagopal V, Meduri C, et al. Early Experience with New Transcatheter Mitral Valve Replacement. JACC 2018. 71(1):12-21.

11. Muller DW, Sorajja P, Duncan A, et al. 2-Year Outcomes of Transcatheter Mitral Valve Replacement in patients with severe symptomatic mitral regurgitation. JACC 2021. 78(19): 1847-1859.

12. Yoon SH, Whisenant BK, Bleiziffer S, et al. Outcomes of Transcatheter Mitral Valve Replacement for degenerated bioprostheses, failed annuloplasty rings, and mitral annular calcification. Eur Heart J 2019. 40(5): 441-451.

13. Mack M, Carroll JD, Thourani V, et al. Transcatheter Mitral Valve Therapy in the United States: A Report from the STS/ACC TVT Registry. Ann Thorac Surg 2022. 113(1):337-65.

14. Zoccai GB, Abbate A, D'Ascenzo F, et al. Percutaneous Coronary Intervention in Nonagenarians: 
pros and cons. J Geriatr Cardiol 2013. 10:82-90.

15. D'Ascenzo F, Bollati M, Clementi F, et al. Incidence and Predictors of coronary stent thrombosis: Evidence from an international collaborative meta-analysis including 30 studies, 221,066 patients, and 4276 thromboses. Int J Cardiol 2013. 167(2): 575-84.

16. Lichtman JH, Kapoor R, Wang Y, et al. Temporal trends of outcomes for nonagenarians undergoing coronary artery bypass grafting, 1993 to 1999. Am J Cardiol 2007. 100:1630-1634.

17. Overtchouk P, Piazza N, Granada J, et al. Advances in transcatheter mitral and tricuspid therapies. BMC Cardiovasc Disorders 2020.20:1-10.

18. Otowa K, Kohsaka S, Sawano M, et al. One-year Outcome after Percutaneous Coronary Intervention in nonagenarians: Insights from the J-PCI OUTCOME Registry. Am Heart J 2022. 246:105-116.

19. Cepas-Guillen PL, Echarte-Morales J, Caldentey G, et al. Outcomes of Nonagenarians with Acute Coronary Syndrome. JAMDA 2022. 23:81-86. 